\title{
Improved calibration of DD neutron counters by activation of two indium disks in the Frascati 1-MJ plasma focus
}

Cite as: Review of Scientific Instruments 56, 1087 (1985); https://doi.org/10.1063/1.1138231

Published Online: 04 June 1998

\section{Edmondo Pedretti, Jean Pierre Rager, and Vittorio Merlo}

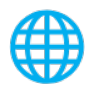

\section{ARTICLES YOU MAY BE INTERESTED IN}

Neutron activation system using water flow for ITER

Review of Scientific Instruments 74, 1735 (2003); https://doi.org/10.1063/1.1534398

\section{Challenge us.}

What are your needs for periodic signal detection?

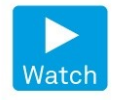

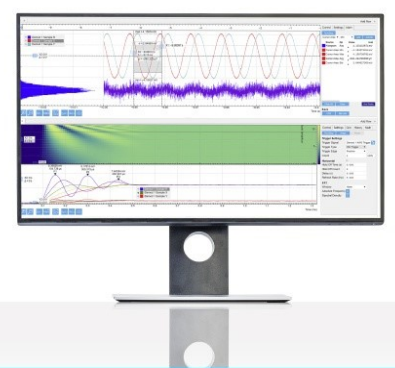

Zurich - Instruments 


\title{
mproved callbration of DO neutron counters by activation of two indium disks in the Frascati $1-M J$ plasma focus
}

\author{
Edmondo Pedretti \\ ENEA, Dip.TIB/FICS, CRE Casaccia, CP 2400, Roma 00100, Italy \\ Jean Pierre Rager ${ }^{\text {a) }}$ and Vittorio Merio ${ }^{\text {() }}$ \\ Assoc. EURATOM-ENEA sulla Fusione, CRE Frascati, CP 65, 00044 Frascati, Roma, Italy
}

(Presented on 19 September 1984)

\begin{abstract}
Three Geiger counters made sensitive to DD neutrons by paraffin covers with interposed $\mathrm{Ag}$, ${ }^{109} \mathrm{Ag}$, and $\mathrm{Rh}$ foils were calibrated by exposing them to $50 \mathrm{PF}$ (plasma focus) shots and by recording counts while simultaneously irradiating two indium disks placed on the PF vacuum vessel. The "polar" disk came out to be about three times more activated than the "equatorial" disk, which was interpreted by assuming an extended focus instead of a point-like one as used in a previous work. The utilized formalism is reported and some minor discrepancies with counters calibration by removal of an Am/B source are discussed. Further refinements of the activation method are suggested.
\end{abstract}

\section{INTRODUCTION}

In a previous work ${ }^{1}$ we described the calibration of a silveractivated GM counter performed by exposing it to a series of plasma focus (PF) shots and recording counts while simultaneously irradiating one indium disk placed close to the focus in a side-on position. In that work, in which the counter was calibrated by other methods, a dispersion of about $15 \%$ was found among the various techniques. As a consequence, improvements were considered to further reduce the discrepancies. In this paper we describe an experiment which was carried out to calibrate three GM counters (activated with natural $\mathrm{Ag},{ }^{109} \mathrm{Ag}$, and $\mathrm{Rh}$ foils) by activation of two indium disks located one in side-on position (equatorial disk) and the other in end-on position (polar disk). The experimental results, incompatible with a point-like focus, were interpreted in terms of a cylindrical focus and of extended disks by a formalism which is shortly outlined. In addition, we made the calibration of the three neutron counters by removal of an Am/B neutron source. Calibration constants determined by both methods are reported and discussed. Finally, possible further refinements of the activation technique are pointed out.

\section{DESCRIPTION OF THE EXPERIMENT}

The Frascati 1-MJ plasma focus machine has been fully described in Ref. 2. The Ag-activated neutron counter used for this work is the same counter shown in Fig. 2 of Ref. 1; the other two counters considered in this paper differ from the first one in the activating foil only, which was ${ }^{109} \mathrm{Ag}$ in the second counter and $\mathrm{Rh}$ in the third one. Fast neutrons coming from the PF, after having been thermalized in the paraffin cover, activate the metallic foils according to the reactions

$$
{ }^{109} \mathrm{Ag}(n, \gamma){ }^{110} \mathrm{Ag} \frac{\beta^{-}}{24.6 \mathrm{~s}}{ }^{110} \mathrm{Cd},
$$

$$
\begin{aligned}
& { }^{107} \mathrm{Ag}(n, \gamma){ }^{108} \mathrm{Ag} \frac{\beta^{-}}{144.6 \mathrm{~s}}{ }^{108} \mathrm{Cd}, \\
& { }^{103} \mathrm{Rh}(n, \gamma)<{ }^{104 \mathrm{~m}} \frac{I T}{4.4 \mathrm{~min}}{ }^{104 \mathrm{~g}} \mathrm{Rh} \frac{\beta^{-1}}{42 \mathrm{~s}}{ }^{104} \mathrm{Pd} \\
& { }^{104 \mathrm{~g}} \mathrm{Rh} \frac{\beta^{-\cdots}}{42 \mathrm{~s}}{ }^{104} \mathrm{Pd}
\end{aligned}
$$

Isotopic composition of the ${ }^{109} \mathrm{Ag}$ foil was determined by irradiating a sample in the Casaccia Triga fission reactor. The subsequent activation analysis gave the following composition: $98 \%$ of ${ }^{109} \mathrm{Ag}$ and $2 \%$ of ${ }^{107} \mathrm{Ag}$ with an accuracy better than $10 \% .^{3}$

The indium disks $(2.8 \mathrm{~cm}$ diameter and $0.2 \mathrm{~cm}$ thickness) were positioned within stainless-steel penetrating cans, as shown schematically in Fig. 1, and kept in place by appropriate disk holders (for other details see Fig. 3 of Ref. 1). The equatorial disk and the polar disk were located at distances of 16 and $14.5 \mathrm{~cm}$, respectively, from the geometrical center O. Temperature of the polar disk was monitored by a thermocouple inserted into the vertical can. The neutron counters were positioned on the equatorial plane at about

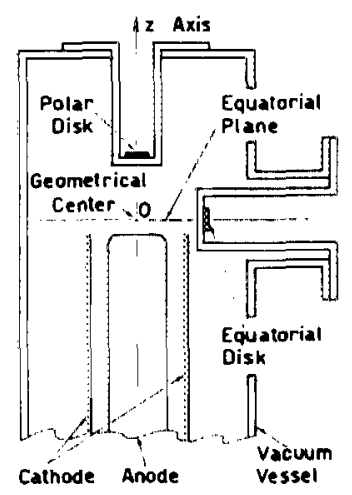

FIG. 1. Schematic arrangement of the irradiation equipment (not to scale). 
$350 \mathrm{~cm}$ from the geometrical center $O$.

The PF machine was operated with electrode geometry of the Mather-type and in a working regime optimized for neutron production $(25 \mathrm{kV}, 390 \mathrm{~kJ}, 6$ torr). The experiment consisted of the performance of 50 shots distributed over a 5 $h$ interval, as shown by Fig. 2 in which vertical bars represent beta counts $G_{12 j}$ taken by the above specified three counters from $T_{1}=100$ to $T_{2}=200 \mathrm{~s}$ after each $\operatorname{shot}(j=1,2, \ldots, 50)$, according to the procedure described in Ref. 1. Deuterium gas was changed after each shot; the above-mentioned thermocouple showed that polar disk temperature never exceeded $90{ }^{\circ} \mathrm{C}$ (indium melting point: $156^{\circ} \mathrm{C}$ ). Activities of the indium disks right after 50 shots were measured by the equipment and by the procedure described in Ref. 1 . The results came out to be $A_{\mathrm{EQ}}=(963 \pm 96) \mathrm{dis} / \mathrm{s}$ for the equatorial disk, and $A_{\mathrm{POL}}=(3173 \pm 317) \mathrm{dis} / \mathrm{s}$ for the polar disk.

\section{DETERMINATION OF CALIBRATION CONSTANTS}

In Ref. 1 it was shown that the calibration constant $K$, which relates the beta counts $G_{12 j}$ to the neutron yield $Y_{j}$ of the $j$ th shot through the formula

$$
Y_{j}=K G_{12 j}, \quad(\text { with } j=1,2, \ldots, N),
$$

can be expressed, in the case of the single equatorial disk, as

$$
K=\left(4 \pi r_{0}^{2} A\right) /\left(N_{T} \bar{\sigma} \lambda C \sum_{j=1}^{N} G_{12 j} \exp \left(-\lambda T_{j}\right)\right),
$$

where $r_{0}$ represents the distance of the indium disk from the focus and $C$ is a factor which is equal to 1 when the focus and the disk are assumed to be point-like.

Now, with three counters and two indium disks one has to do with six calibration constants which should lead to the same neutron yield for any PF shot. In addition, for a given neutron counter the two calibration constants $K_{\mathrm{EQ}}$ and $K_{\mathrm{POL}}$, obtained from the equatorial and polar indium disks, should be the same, i.e., one should have

$$
K_{\mathrm{EQ}} / K_{\mathrm{POL}}=1 \text {. }
$$

Contrary to this requirement, it was found that by assuming point-like focus and disks (with the focus located on

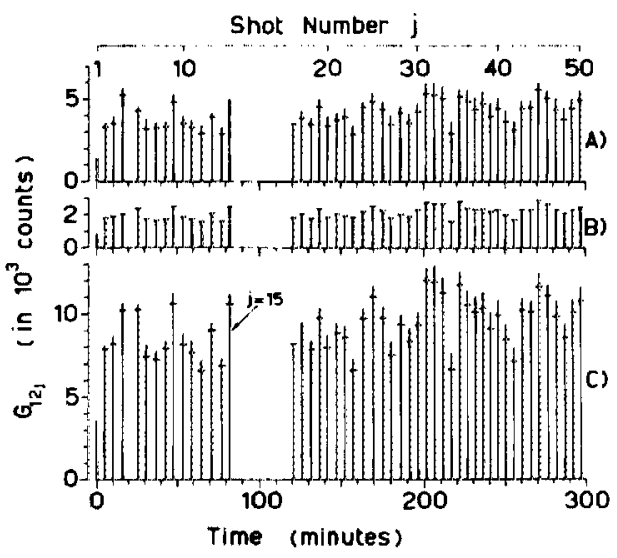

Fig. 2. Beta counts $G_{12 j}$ vs time after the first shot. Upper ends of vertical bars represent uncorrected counts; cross marks near upper ends represent counts corrected for residual foil activity due to previous shots. (a) Ag-activated counter; (b) ${ }^{109} \mathrm{Ag}$-activated counter; (c) Rh-activated counter. the geometrical center $\mathrm{O}$ ), this ratio comes out to be about 0.382 , which is approximately equal to the ratio of the activities $A_{\mathrm{EQ}}$ and $A_{\mathrm{POL}}$.

To overcome this inconsistency and to take into account experimental evidence on the extended localization of the neutron source, ${ }^{4}$ we have abandoned the simplified assumption of a point-like focus. Furthermore, we have also considered extended indium disks.

The geometry used for this new approach is shown in Fig. 3, in which $d V=r d \phi d r d z$ is the generic volume element of the focus and $d \mathrm{a}=\rho d \delta d \rho$ is the generic surface element of the indium disk. With this arrangement it can be shown that the coefficient $C$ appearing in $\mathrm{Eq}$. (5) is expressed by

$$
C=r_{0}^{2} \int_{V} d V \int_{\mathrm{a}}\left[\beta(\gamma) / X^{2}\right] d \mathrm{a} /(V \mathrm{a})
$$

where $X$ is the distance of $d a$ from $d V, \gamma$ is the incidence angle for the neutrons reaching $d \mathbf{a}$ from $d V$, and $\beta(\gamma)$ is a reduction factor taking into account removal of neutrons in the structural materials existing between focus and indium disk. Expressions for $X$ and $\gamma$ can be derived from the geometry of Fig. 3.

Obviously, with the geometry of Fig. 3 one calculates the coefficient $C_{\mathrm{EQ}}$ for the equatorial disk. With a similar geometry one can calculate the coefficient $C_{\text {PoL }}$ for the polar disk. Entering Eq. (6) with $K_{\mathrm{EQ}}$ and $K_{\mathrm{POL}}$, as derived from Eq. (5), one obtains that the coefficients must fulfill the condition

$$
\begin{aligned}
& C_{\mathrm{POL}} / C_{\mathrm{EQ}}=\left(A_{\mathrm{POL}} N_{T, \mathrm{EQ}} r_{0, \mathrm{POL}}^{2} \bar{\sigma}_{\mathrm{EQ}}\right) / \\
& \left(A_{\mathrm{EQ}} N_{T, \mathrm{POL}} r_{0, \mathrm{EQ}}^{2} \bar{\sigma}_{\mathrm{POL}}\right) .
\end{aligned}
$$

Concerning the indium cross sections we have used neutron spectra measured by nuclear emulsion plates located end-on and side-on, ${ }^{5}$ to obtain $\bar{\sigma}_{\mathrm{EQ}}=(0.275 \pm 0.028) b$ and $\bar{\sigma}_{\mathrm{POL}}=(0.303 \pm 0.031) \mathrm{b}$. The masses of our two indium disks were found to be equal within $0.1 \%$; as a consequence the numbers of target nuclei are also equal and can be dropped. Entering Eq. (8) with the remaining known values gives

$$
C_{\mathrm{POL}} / C_{\mathrm{EQ}} \simeq 2.455 \text {. }
$$

As a preliminary representation of the extended focus we have assumed a cylindrical volume coaxial with the $z$ axis and having radius $R$ and height $H_{1}+H_{2}$ as shown by the dashed contour in Fig. 3. From the experimental data on the localization of the neutron source, ${ }^{4}$ we have put as a first

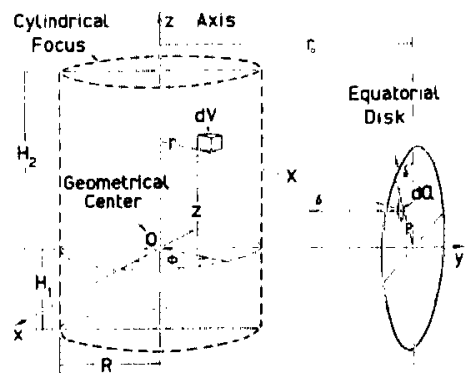

FIG. 3. Geometry used for extended focus and equatorial indium disk. 
Table I. Calibration constants $K$ of neutron counters.

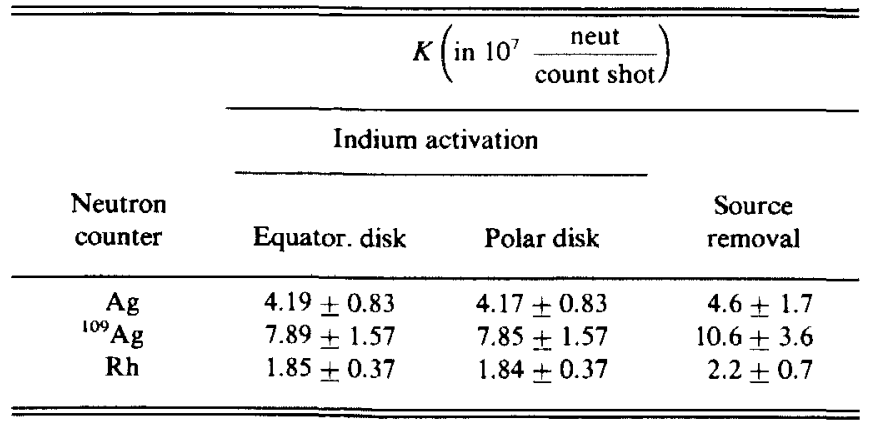

hypothesis $R=H_{1}=1 \mathrm{~cm}$. Since the maximum incidence angle is about $33^{\circ}$ (in the case of the equatorial disk) we have used, as a first approximation, the assumption $\beta(\gamma)=\cos \gamma$. The integral appearing in Eq. (7) was determined numerically by the Simpson method. The free parameter $\mathrm{H}_{2}$ was looked for by a trial and error procedure aimed at satisfying condition (9). The results were $H_{2}=8.4 \mathrm{~cm}, C_{\mathrm{EQ}}=0.8937$, and $C_{\text {POL }}=2.201$. Then, from Eq. (5) we obtained the six calibration constants reported in Table I, which also includes three calibration constants determined by the removal of a 10-Ci Am/B neutron source. The formalism to be used when applying the source removal method to a Agactivated counter was derived in Ref. 1; for ${ }^{109} \mathrm{Ag}$ - and $\mathrm{Rh}$ activated counters the pertinent formalisms, derived from reactions (2) and (3), respectively, are reported in Ref. 6.

\section{DISCUSSION AND CONCLUSIONS}

Before the experiment described in Sec. I, we had carried out a preliminary test consisting of 41 shots, in which distribution of beta counts and activities of indium disks had characteristics quite similar to those reported in Sec. I. This similarity of experimental results allows us to be confident of their reproducibility.

Concerning the calibration constants shown in Table $\mathbb{l}$, we note that they agree within experimental errors and that the calibration constants determined by indium activation appear to be systematically somewhat smaller and to have less dispersion than those obtained by source removal. This difference may be attributed to various causes, the most important of which seems to reside in the $\mathrm{Am} / \mathrm{B}$ neutron source whose intensity $\left(5 \times 10^{6} \pm 20 \%\right.$ neut/s) is not very well known (besides being too low for the source removal method ') and whose angular neutron emission, not measured, may appreciably differ from that of the focus. Another cause may be the difference between the neutron spectra existing in the indium disks locations and the neutron spectra measured by end-on and side-on nuclear plates, whose positions were somewhat farther away with respect to the indium disks locations. And finally, a systematic error of the indium activation method is certainly represented by the coarse assumption of a cylindrical focus and of a uniform neutron emission, which implies flat profiles of ion density and ion temperature. In this connection it appears possible to refine the approach by using more elaborate models of the extended focus, such as the one considered in Ref. 7.

As far as the details of the adopted model are concerned it may be interesting to observe that Eq. (7) can be improved by the insertion of two factors, $g(\mathbf{r})$ and $f(\Omega)$ (possibly based on experimental evidence), describing the dependence of neutron emission on the position $\mathrm{r}$ of the focus element $d V$ and on the direction $\boldsymbol{\Omega}$ along which neutrons are emitted from $d V$. Another small improvement can be obtained by using a reduction factor $\beta(\gamma)$ determined experimentally.

In the end, it may be worthwhile to notice that Eq. (5) implies constancy of volume and shape of the extended focus during $N$ shots, a condition that certainly does not occur. This inconvenience, which can cause a minor inaccuracy, can be eliminated if the indium activity is high enough to be measured on a single-shot basis $(N=1)$ by using a $\mathrm{Ge}(\mathrm{Li})$ detector instead of a $\mathrm{NaI}$ crystal, an improvement which would also simplify the overall procedure.

As concluding remarks it seems reasonable to state that the accurate determination of the neutron yield is still an issue which offers room for improvements and that the indium activation method looks promising especially because it makes use of the neutrons produced by the plasma itself, without need of an external neutron source. The main difficulty of the indium activation method appears to be the choice of the most appropriate plasma model.

\section{ACKNOWLEDGMENTS}

The authors want to thank $M$. Tacchi for operating the PF, E. Cafaggi for providing penetrating cans and disks holders, A. Moauro for activation analysis, and P. Moioli and $\mathbf{R}$. Scafè for measuring indium activities.

") Present address: Commission of the European Communities, Directorate General XII, Fusion Programme, 1049 Brussels, Belgium.

bl Present address: JET Joint Undertaking, Abingdon, United Kingdom.

'A. Gentilini, J. P. Rager, K. Steinmetz, M. Tacchi, D. Antonini, B. Arcipiani, P. Moioli, E. Pedretti, and R. Scafè, Nucl. Instrum. Methods 172, 541 (1980).

${ }^{2}$ A. Bernard, P. Cloth, H. Conrads, A. Coudeville, G. Gourlan, A. Jolas, Ch. Maisonnier, and J. P. Rager, Nucl. Instrum. Methods 145, 191 (1980). ${ }^{3}$ A. Moauro (private communication).

${ }^{4}$ K. Steinmetz, K. Hübner, J. P. Rager, and B. V. Robouch, Nucl. Fusion 22, 25 (1982).

SJ. P. Rager, Proceedings of the Vth Course on Unconventional Approaches to Fusion, Erice, 16-25 March 1981 (Plenum, New York, 1981).

${ }^{6} \mathrm{~V}$. Merlo. Thesis, University of Roma, 1983.

${ }^{7}$ J. P. Rager, Yu. Alexeev, J. Appelt, J. S. Brzosko, J. Erhardt, G. Gourlan, K. Hübner, L. Ingrosso, M. Kazeev, P. Kirchesch, J. Klobukowska, H. Kroegler, S. Podda, B. v. Robouch, and K. Steinmetz, Report 82.40/cc, Associazione EURATOM-ENEA sulla Fusione, CRE Frascati, August 1982. 